\title{
Optimal Placement of SVC using NSGA-II
}

\author{
Shishir Dixit ${ }^{1}$, Laxmi Srivastava ${ }^{2}$ and Ganga Agnihotri ${ }^{3}$ \\ ${ }^{1,2}$ Electrical Engineering Department \\ MITS, Gwalior, India \\ ${ }^{3}$ Electrical Engineering Department \\ MANIT, Bhopal, India \\ 1shishir.dixit1@gmail.com,2srivastaval@hotmail.com,3ganga1949@gmail.com
}

\begin{abstract}
Improving voltage stability, reducing real power loss (PL) and voltage deviation (VD) are the most important tasks in the operation of electrical power systems. Voltage instability and voltage collapse are the severe problems which may take place because of deficit reactive power at load buses due to increased loading or contingencies. In this paper, the problem of obtaining optimal location and size of SVC is formulated as true multi-objective optimization problem for simultaneous minimization of the two objectives namely real power losses and load bus voltage deviation. The two algorithms real coded genetic algorithm (RCGA) and non-dominated sorting genetic algorithm-II (NSGA-II) with a feature of adoptive crowding distance have been used for solving nonlinear constrained multi-objective optimization problem. Both the algorithms have been used for obtaining optimal location and sizing of SVC. Voltage security of the power system has also been analyzed separately for all placement of SVC to ensure secure operation of the system. The proposed approaches have been implemented on IEEE 30-bus test system. The simulation results of the two algorithms have been compared for solution quality, computational complexity and computational time. It has been found that NSGA-II presents better performance in solving multi-objective optimization problem and also in obtaining a diverse set of solutions which converge near the true Pareto-optimal front. The simulation results of NSGA-II have also been presented to exhibit the capabilities of the algorithm to generate well-distributed Pareto-optimal front.
\end{abstract}

Keywords: Multi-objective optimization; NSGA-II; RCGA; SVC; real power losses $(P L)$; voltage deviations $(V D)$

Introduction

In the last few decades, several major blackouts which have been reported worldwide e.g. in Italy, France, Great Britain, Japan, WSCC in USA, etc. were interrelated to voltage collapse. Thus, problems of voltage collapse in power system networks have been main concern for electric power utilities all over the world [1]. The heavily loaded electric power network and/or deficient local reactive power sources may result in voltage instability [2].

The remarkable efforts have been done to find the different ways to ensure the power system security and voltage stability. It is concluded that for the power system networks which operates very close to the steady-state stability limit and may result in voltage instability and/or voltage collapse, use of flexible alternative current transmission system (FACTS) devices can prove a good choice for improving the static voltage stability margin [3]. FACTS family include thyristor controlled series compensator (TCSC), static Var compensator (SVC), unified power flow controller (UPFC), static synchronous compensator (STATCOM) etc [4-8]. Application of FACTS results in power flow control smoothly and rapidity [9]. The benefit provided by FACTS devices are highly affected by certain aspect which mainly include their location and sizing [10]. Placement of FACTS in 
power system network is decided by extensive investigation of some important technical factors including steady-state stability, voltage stability, small signal stability etc. along with the practical factor such as installation conditions, cost etc. [3].

In the literature, worldwide researchers have shown their dedicated interest for obtaining the optimal location and sizing of FACTS devices in power systems and suggested various methodologies and criteria for the same. For voltage stability enhancement optimal location of SVC and other shunt compensation devices are reported [11]. The authors suggested the modal analysis method in the vicinity of the point of collapse of the test system. In [12] optimal locations, sizing and types of FACTS devices namely SVC and TCSC are suggested for asset utilization. The proposed approach is based on combining static and dynamic procedure which also incorporates an optimal power flow, power flow continuation, and an analysis of eigen value. The sensitivity-based method has been proposed for optimal placement of FACTS devices to enhance system transmission capacity, power of an electric market pool and contractual dispatches [13]. The optimal placement of the SVC for simultaneous improvement in the static and dynamic voltage security of the electric power system is suggested by sensitivity based approach [14]. In [15], optimal location and numbers of TCSC have been searched for solving the problem of the deregulated electricity markets by applying mixed integer nonlinear programming approach.

Apart from mathematical modeling as discussed above, number of population based heuristic methods have also been applied for finding optimal location and sizing of FACTS devices in power system networks. Farsangi et al. [10] applied particle swarm optimization (PSO) and genetic algorithms (GA) for improving voltage profile and minimizing power losses by placement of SVC. In [9] minimization of cost of installation and improved system loadability were achieved by searching optimal location of multi-type of FACTS devices, namely SVC, TCSC, and UPFC using PSO. The two objective functions were converted into a single objective function. In [16] GA was implemented for optimization of four types of FACTS devices namely SVC, TCVR, TCSC, TCPST in order to improve loadability of the system. The optimizations were carried out for three parameters namely locations, types, and settings of these devices. In [17], the genetic algorithm has been implemented for cost minimization with finding optimal types and sizing of FACTS devices namely the SVC, TCPST, TCSC, UPFC. The cost also includes the investment costs of the FACTS devices and the bid of the market participants. Here also, the two objectives were converted into a single objective function.

In [18], a genetic algorithm-based method was applied to find out the optimal location and number of UPFC devices in an assigned power system network to maximize system capabilities, social welfare and for satisfying contractual requirements of an open electric power market. The cooperation between all these objectives functions was calculated using fuzzy logic. The optimal placement of SVC is done in the electric power system using genetic algorithm, particle swarm optimization, simulated annealing (SA) and artificial immune system (AIS) [19, 20]. In [21] SA has been used for finding locations, types of Var sources devices and their sizing considering various loading conditions. The overall cost of the Var sources i.e. installation cost, purchase cost, and cost of energy losses are minimized considering operational constraints. GA is applied for placement of only one SVC to minimize power loss, voltage deviation and cost within a power system. In [22] AIS based approach is applied to improve voltage profile and minimize losses by optimally placement of SVC. Multi-objective GA is used to find optimal locations of FACTS devices in a power system [23]. In [24] for improving power system security, optimal placement of FACTS devices are determined using harmony search algorithm. A novel global harmony search (NGHS) algorithm is also implemented on same problem and results are compared that obtained using harmony search (HS) algorithm and PSO for enhancing voltage stability and voltage profile, minimizing real power losses and total investment cost with 
optimal placement of SVC and STATCOM. The NGHS results in providing better accuracy, higher speed to find solution in comparison of HS and PSO [25]. A new big bang-big crunch (BB-BC) optimization algorithm is used for finding optimal location and sizing of FACTS for improving voltage stability limit, voltage profile and loss minimization under normal as well as for line outage contingency conditions. The BB-BC algorithm similar to PSO involves less operators, thus it is also straightforward to execute for optimization problem of power system. The BB-BC demonstrates superior performance and convergence characteristics in compare of PSO [26]. In [27] for enhancing voltage profile and minimizing power loss, voltage deviation and size of SVC, the optimal location and size of SVC has been obtained using GA and critical contingency. For improving voltage stability of the power system the optimal location and size of TCSC has been find out using PSO and PSO-TVAC. It has been found that PSO-TVAC provides faster convergence and better solution quality as compare to PSO [28].

In general, from the previous works reported for FACTS placement in power system, it can be concluded that most of the problem for obtaining optimal location and sizing of FACTS devices are formulated as problem of mono-objective optimization. These monoobjective problems optimize a single objective for FACTS placement. In some of the optimization problems conversion of a number of objectives to a single objective is achieved by combining them, or by using fuzzy inference system. The multi-objective problem formulation for optimal placement of FACTS placement is a latest attempt in this field.

In this paper investigation of the optimal location and sizing of SVC is formulated as a real multi-objective optimization problem by combining two objective functions namely real power loss and voltage deviation. The work proposed for obtaining the optimal location and sizing of SVC has been carried out in two steps. In first part of the proposed methodology, investigation of single critical line outage contingencies has been carried out. After that real coded genetic algorithm (RCGA) and non-dominated sorting genetic algorithm-II (NSGA-II) has been applied one by one to solve the formulated optimization problem. Finally, the optimization techniques have been compared for their execution time and solution quality.

The organization of the paper is as follows: Section 2 presents problem formulation, Section 3 discusses problems of multi-objective optimization, a fuzzy based approach for the best compromise solution is discussed in Section 4. In Section 5 and Section 6 brief introduction of RCGA and NSGA-II are presented. The simulation results are discussed in Section 7. Finally, conclusion and future scope of the work is given in Section 8.

\section{Problem Formulation}

In this paper, for optimal placement of SVC single line outages in a power system are considered as contingencies. The severity of a contingency (i.e. single line outage) is evaluated using Voltage Power Index (VPI) [29] as:

$$
V P I=\sum_{i=1}^{N B}\left(\Delta\left|V_{i}\right| / \Delta\left|V_{i}^{\max }\right|\right)^{2 m}
$$

where, $\Delta\left|V_{\mathrm{i}}\right|$ is absolute difference between the voltage magnitude under line outage and base case condition; $\Delta\left|V_{i}^{\max }\right|$ is bus voltage magnitude chosen by the utility engineers to indicate how much acceptable limit should be for an outage case; NB is number of buses in the system. In this paper, the value of the exponent $m$ has been taken as $2, \Delta\left|V_{i}^{\max }\right|$ has been considered as 0.2 p.u and NB has been taken as 30 respectively. 
Minimization of real power loss

The real power loss (RPL) as first objective function $F_{1}$ is defined as:

$$
P_{L}=\sum_{k=1}^{N T L}\left[g_{k}\left[V_{i}^{2}+V_{j}^{2}-2 V_{i} V_{j} \cos \left(\delta_{i}-\delta_{j}\right)\right]\right.
$$

where, $N T L$ and $g_{k}$ are the number of transmission lines and conductance of $k^{\text {th }}$ line; at the both ends of $k^{\text {th }}$ line the bus voltages are $V_{i} \angle \delta_{i}$ and $V_{j} \angle \delta_{j}$, respectively .

\section{Minimization of Voltage Deviations}

The load bus voltage deviation (VD) as second objective function $F_{2}$ is defined as:

(3)

$$
V D=\sum_{k=1}^{N L}\left|\left(V_{k}-V_{k}^{r e f}\right)\right|
$$

In this paper, $V_{k}^{r e f}$ is taken as $1.0 \mathrm{p}$.u. In a power system, unless specified, it is accustomed to maintain the load bus voltages within $\pm 5 \%$ of its nominal value.

Multi- Objective function

The multi-objective function for the optimization problem is obtained by combining all objective functions mentioned above as $\mathrm{F}(u, v)$ :

$$
\begin{aligned}
& F(u, v) \\
& =w_{1} \mathrm{~F}_{1}(u, v) \\
& +w_{2} \mathrm{~F}_{2}(u, v)
\end{aligned}
$$

here, weighted sum method is used to formulate multi-objective function $\mathrm{F}(u, v)$ by combining objective functions of real power loss and load bus voltage deviation. The two objective functions are combined to form single multi-objective function using weighing factor $w$. In this study $w_{1}$ and $w_{2}$ are varied randomly between 0 and 1 , and $w_{2}=1-w_{1}$. Now, the optimization is performed for minimizing the multi-objective function $\mathrm{F}(u, v)$ considering equality and inequality constraints.

\section{Constraints}

\section{Equality Constraints}

The equality constraints are the typical load flow equations given as follows:

(5)

$$
\left.P_{G i}-P_{D i}=V_{i} \sum_{j=1}^{N B} V_{j}\left[G_{i j} \cos \left(\delta_{i}-\delta_{j}\right)+B_{i j} \sin \left(\delta_{i}-\delta_{j}\right)\right)\right]
$$

$$
\text { for } i=1, \ldots \ldots ., N B
$$

$Q_{G i}-Q_{D i}=$

$V_{i} \sum_{j=1}^{N B} V_{j}\left[G_{i j} \sin \left(\delta_{i}-\delta_{j}\right)-\right.$

$\left.B_{i j} \cos \left(\delta_{i}-\delta_{j}\right)\right]$

for $i=1, \ldots \ldots ., N B$

where, $P_{G i}, Q_{G i}$ represents real and reactive powers of the generator and $P_{D i}, Q_{D i}$ represents the active and reactive power of load at bus $i$ respectively; $G_{i j}$ and $B_{i j}$ the transfer conductance and susceptance of the line between bus $i$ and bus $j$, respectively.

Inequality Constraints 
Inequality constraints are the upper and lower limits of reactive power of a generator. The reactive power of $i^{t h}$ generator must lie within its minimum $\left(Q_{g_{i}}^{\min }\right)$ and maximum $\left(Q_{g_{i}}^{\max }\right)$ limits as:

$$
Q_{g_{i}}^{\min } \leq Q_{g_{i}} \leq Q_{g_{i}}^{\max } \quad i=1,2 \ldots N G
$$

\section{Multi-objective Optimization}

In general, real-world problems are noncommensurable having contending and contradictory nature of objectives. Such problems necessitate simultaneous optimization of different objectives. The optimization results of these multi-objective optimization problems are a set of solutions rather than a single solution. Considering all the objectives at the same time no single solution can be considered better than any other solution. These optimal solutions are known as Pareto-optimal solutions. The multi-objective optimization problem statements are a combination of several objective functions and also include several equality and inequality constraints. Thus, multi-objective optimization problem can be expressed as:

$$
\text { Minimize } F_{I}(x) \quad I=1, \ldots, N_{o b j}
$$

Subjected to

$$
\left\{\begin{array}{c}
g_{k}(x)=0 \quad \text { where } k=1 \ldots . K \\
\hbar_{l}(x) \leq 0 \quad \text { where } l=1 \ldots . . L
\end{array}\right.
$$

where $F_{I}$ is the $I^{\text {th }}$ objective function and $x$ is decision vector that represents a solution. $K$ and $L$ are number of equality and inequality constraints.

\section{Best Compromise Solution}

The decision maker uses the approach mentioned below for obtaining one solution which is the best compromise solution, from the Pareto-optimal front of non-dominated solution [23]. The $i^{\text {th }}$ objective function $f_{i}$ having membership function $\mu_{i}$ can be define as :

$$
\mu_{i}=\left\{\begin{array}{lc}
1 & f_{i} \leq f_{i}^{\text {min }} \\
\frac{f_{i}^{\text {max }}-f_{i}}{f_{i}^{\max }-f_{i}^{\text {min }}} & f_{i}^{\text {min }}<f_{i}<f_{i}^{\text {max }} \\
0 & f_{i} \geq f_{i}^{\text {max }}
\end{array}\right.
$$

where for $i^{\text {th }}$ objective function the minimum and maximum value of the objective functions are represented as $f_{i}^{\min }$ and $f_{i}^{\text {max }}$ respectively amongst all non-dominated solutions. The normalized membership function $\mu^{k}$ for any non-dominated solution $k$, is computed as :

$$
\mu^{k}=\frac{\sum_{i=1}^{N_{o b j}} \mu_{i}^{k}}{\sum_{k=1}^{M} \sum_{i=1}^{N_{o b j}} \mu_{i}^{k}}
$$


where $M$ is the number of non-dominated solutions and $N_{o b j}$ is number of objective functions. In this paper $N_{o b j}$ is equal to 2 . The solution which has the highest value of $\mu^{k}$ is considered as the best compromise solution.

\section{Real Coded Genetic Algorithm}

GA is one of the well established heuristic techniques to solve complex optimization problems. GA is also a global search method which search for numerous solutions without knowing solution properties in advance. Real coded genetic algorithm (RCGA) is very similar to binary GA [27, 30-33]. The difference between binary coded GA (BCGA) and RCGA is that variables in RCGA are represented by floating-point numbers instead of bits of zero and ones. The structure of RCGA is very closely resembles human reasoning and thus it is also called as intelligent. While dealing with continuous search space BCGA suffers with some problems. One of the most common problems is Hamming cliffs which is associated with some strings like 01111 and 10000 where conversion to adjacent solution (in real space) needs alteration of a number of bits. Finding arbitrary precision in optimal solution is another common problem of BCGA. Use of floating-point numbers for representation of the problem parameters in RCGA has overcome such difficulties specially when dealing with continuous search space. The structure of RCGA basically includes three main steps: selection, crossover (recombination) and mutation [27, 30].

In this paper, the formulation of SVC placement is done using some parameters which include the location and size of SVC. In a string first value represents location of SVC. It is basically number of load buses where SVC can be installed. The remaining values in the string represent the possible sizing of SVC.

Initial population depends on the number of possible locations (i.e. load buses) for SVC placement $N_{B}, N_{s}$ represents the number of various sizing of SVC and $N_{\text {ind }}$ represents individuals in the population. Any individual is generated in following steps: as a first step a set of $N_{B}$ i.e. number of load buses in system is selected randomly and inserted at the first location of each string. Thereafter, a set of $N s$ representing different sizing of the SVC are randomly chosen. The entire initial population will be attained when the procedure is repeated for $N_{\text {ind }}$ times. The objective function is computed for each individual. In this way, the new generation will be produced by the outcome that have already achieved by the old generation.

In this paper, Roulette wheel selection technique has been used [27, 30]. By selecting probability of crossover $P_{C}$ near to 1 , crossover has been applied between the best selected individuals (i.e. parents) to create a new individuals. The intermediate recombination has been also applied in this paper [27].

All the above mentioned operators have been used to produce the new offspring. The objective function is computed for all of the offspring and some of the best individuals out of them are selected to create new generation. This process is used to compare the best value of objective function and average fitness of new generation; the earlier generation must be at the same level or higher. The flowchart of RCGA is shown in Fig. 1. 


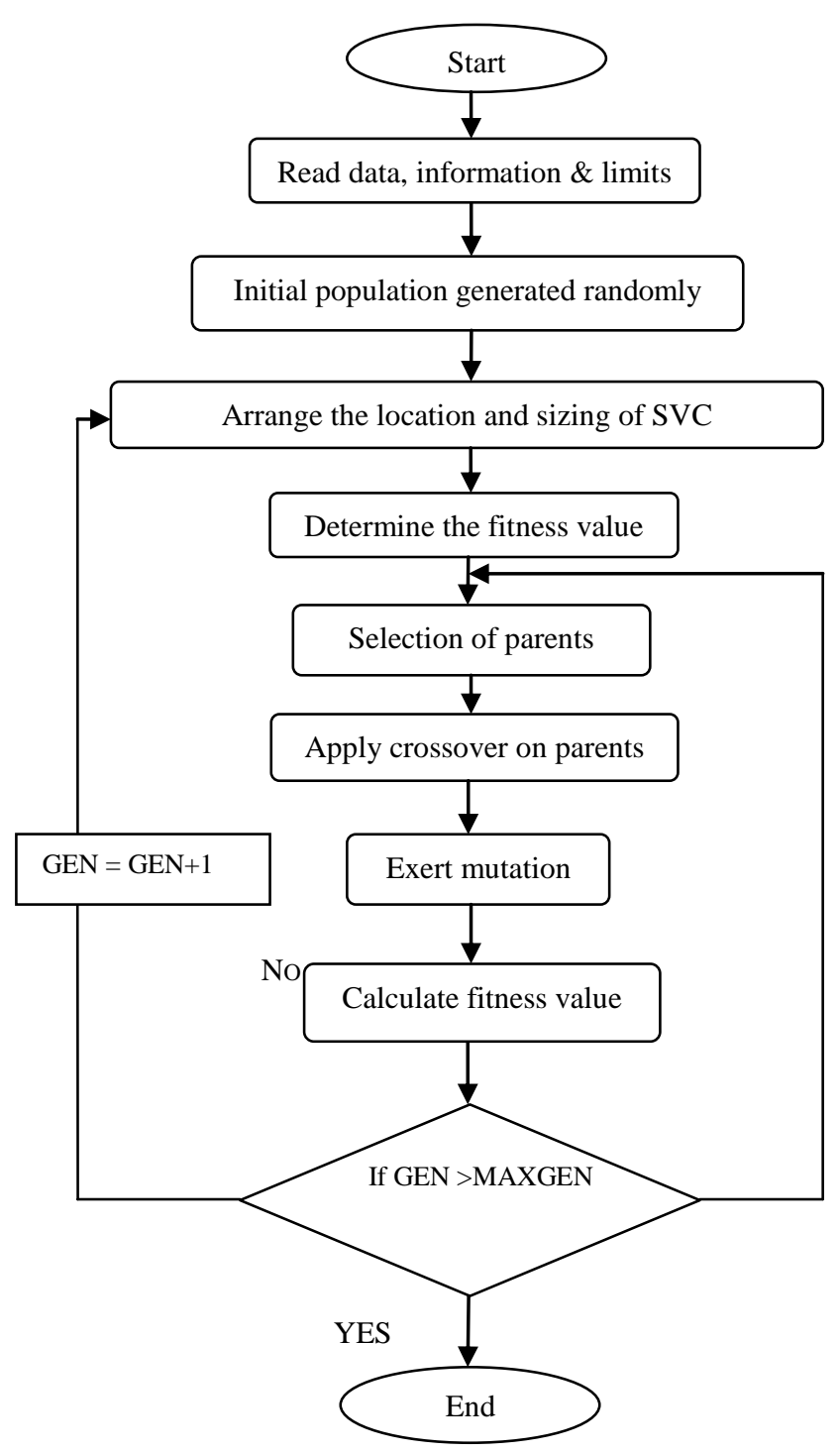

Flowchart of RCGA Implementation

\section{Non-dominated Sorting Genetic Algorithm-II}

The NSGA which was introduced by Srinivas and Deb employs a new method for selection operator which is based on classes of dominance of all the solutions $[29,31,33]$. The limitations of NSGA have been exposed by researchers like uncertainty of finding elitism, computational complexity and also in choosing the value of optimal parameter for sharing parameter. To overcome the limitations of NSGA, an advanced version NSGA-II has been developed which incorporates elitism, better sorting algorithm and need not to choose sharing parameter in advance.

\section{Universal approach}

A brief introduction of some important concepts of algorithm such as non-dominated sorting procedure, evaluation of crowding distance and crowded-comparison operator have been presented here [32,36]: 
(a) Non-dominated sorting procedure: Each solution is compared with all other solutions in the population in order to find its dominance. In this way all dominated solutions are identified to form first non-dominated front. The solutions of the first front are discounted temporarily while obtaining the individuals of the next non-dominated front. This procedure of identification of non-dominated front is repeated until third and higher levels of non-domination are identified.

(b) Evaluation of crowding distance: For evaluating crowding distance, the density of solutions nearby a particular solution in the population, the average distance of two points on either side for this point under consideration is calculated for each of the objectives. The distance thus calculated provides an estimate of the perimeter of the cuboid formed by using the nearest neighbors as the vertices. This is called crowding distance. The crowding distance so calculated is used to sort the population in accordance to magnitude of each objective function in ascending order of magnitude. Subsequently, the boundary populations i.e. the highest and lowest value of objective function are designated as an infinite distance value. The all remaining intermediate populations are assigned with distance value which in fact is equal to the absolute normalized difference in the function values of two adjacent populations. The procedure of calculation is repeated for all other objective functions. The overall crowding distance is considered as addition of all the individual distance calculated for each objective separately. Each objective function is normalized before calculating the crowding distance.

(c) Crowded-comparison operator: The crowded-comparison operator leads to the selection process at the different stages of the algorithm in order to get uniformly well distributed pareto-optimal front. All the population has two important feature nondomination rank and crowding distance. If two populations presenting contradictory nondomination ranks, then the population have the lower rank will be considered better and preferred. If both populations have same front then the population with larger crowding distance is preferred.

\section{Computational Steps}

The computational steps of algorithm are as:

Step 1 Initialization: At first random population of parents $\left(N_{P}^{\prime}\right)$ is created.

Step 2 Non-dominated sorting of parents' population: In order to get first front, the parents' population is sorted in accordance of their non-domination. Starting from very first best non-dominated front all other fronts in the population are sorted and ranked based on non-domination as front no. ' 1 ' for first or best level front ' 2 ' for second or next best level front and so on. The crowding distance of all non-domination level in the populations are calculated and sorted populations in descending order of the crowding distance.

Step 3 Tournament selection: Two individuals are selected randomly and compared for their front number and crowding distance.

Step 4 Crossover and mutation: The simulated binary crossover (SBX) and polynomial mutation [34] has been applied in this work. The probability of crossover is taken as 0.9 and probability of mutation $p_{m}=1 / \mathrm{n}$ where $\mathrm{n}$ is the number of decision variables has been used in this work. The distribution indexes [34] for both crossover and mutation operators have been taken as $g_{c}=20$ and $g_{m}=20$ respectively in this work. The child population of size $N_{P}$ is produced.

Step 5 After Combining both parents and children population the combined population is $2 N_{P}$. 
Step 6 Non-dominated Sorting of combined population: The combined population of parents and children is sorted in accordance to their non-domination and crowding distance. Elitism can surely be ensured as the entire parent and child population members are included. Now, populations of the best non-dominated set $F_{1}$ are supposed to be the best populations in the combined population and must be emphasized more in comparison of any other population in the combined population. The size of $F_{1}$, if smaller than $N_{P}$, it is an indication that all members of the set $F_{l}$ are chosen to form new population. The remaining members for the new population will now be chosen from subsequent nondominated fronts in the order of their ranking. Consequently, population members from the set $F_{2}$ are chosen subsequently, followed by solutions from the set $F_{3}$ and so on. The process is continued until no more sets can be accommodated. The set $F_{n}$ is the last nondominated set ahead of which no extra set can be accommodated. Commonly, the count of solutions in all sets from $F_{l}$ to $F_{n}$ may be larger as compared to population size $N_{P}$. The crowded-comparison operator is used to sort population member of front $F_{n}$ in descending order and all population slots are filled with so chosen the best population member. In this way exactly $N_{P}$ population members are chosen.

Step 7 Stopping rule: The iterative procedure can also be terminated when there is no considerable improvement in the solution. The procedure can also be stop when a given maximum number of generations are reached. In this work, the maximum number of generations has been chosen as the stopping criterion. The stopping criteria are checked. In case the stopping criteria are satisfied go to Step 8 otherwise copy new population to parents' population and go to Step 3 .

Step 8 Select population member of the first front.

Step 9 Stop. 


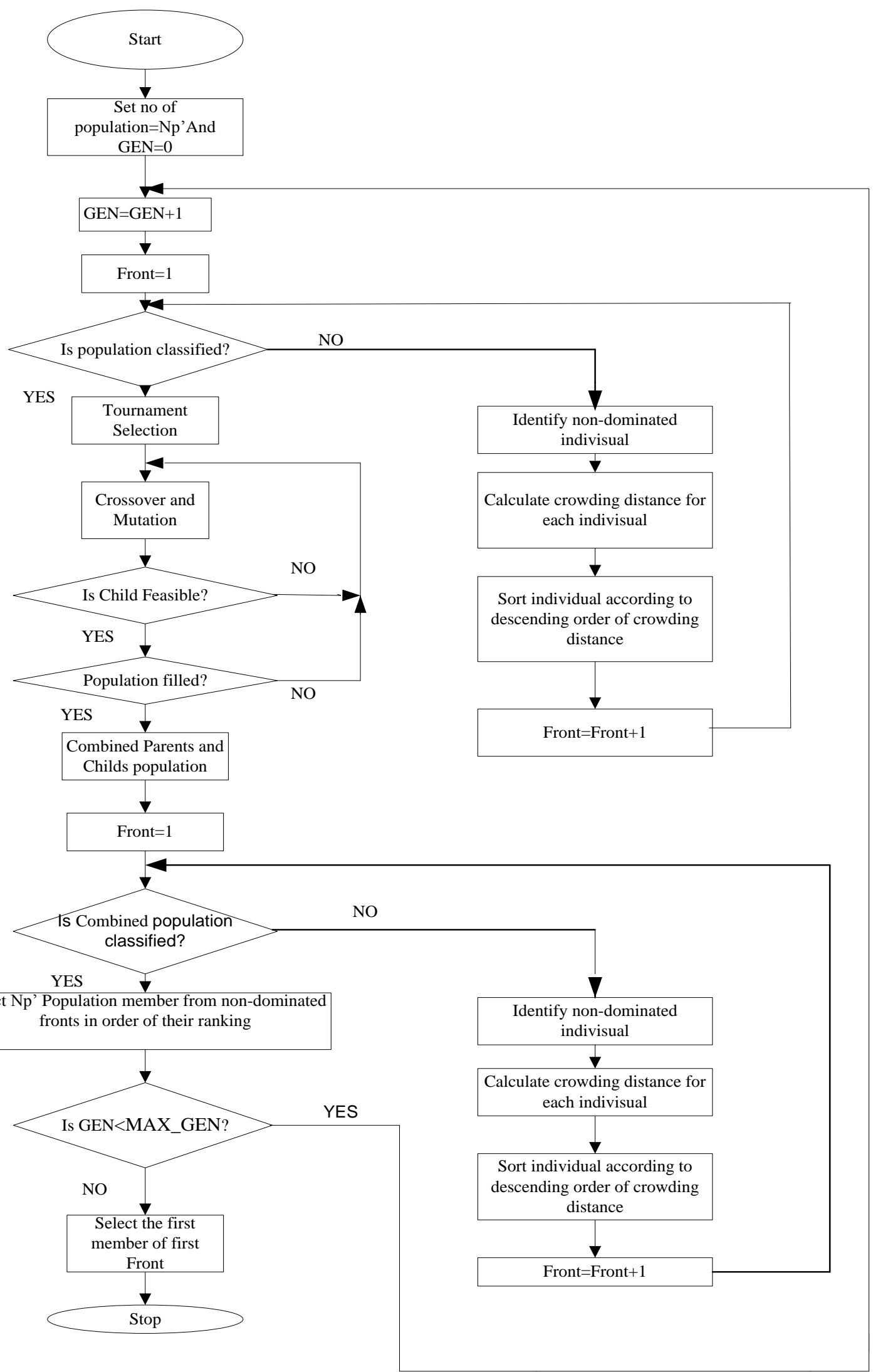

Flowchart of NSGA-II 


\section{SIMULATION RESULTS}

Real coded genetic algorithm and non sorting genetic algorithm-II has been implemented to find optimal location and size of SVC in IEEE 30 bus system [37]. This test system has one slack bus, $5 P V$ buses, $24 P Q$ buses and 41 transmission lines. For optimal placement of SVC, single line outages are considered as contingencies in the test power system and to evaluate the severity of a contingency VPI has been used. It has been observed that NR load flow program converge for only 37 line outages out of 41 line outages. The objective function can be described as a mixed continuous-discrete optimization problem. The placement of SVC is considered as a discreet decision variable. Any of $P Q$ bus from all the $24 P Q$ buses (load buses) may be optimal possible location for SVC placement.

For some of the single line outage contingencies, the voltage magnitude at some buses violated the permissible voltage limit, which is indicated by VPI in this paper as shown in Table 1. As can be observed from Table I, on the basis of VPI, the ranking of critical contingencies obtained as $36,5,15,37,38,25$ and so on. In this paper, only first three most severe contingencies i.e. outage of line nos. 36, 5 and 15 have been considered for SVC placement.

VPI Based Ranking For Line Outage

\begin{tabular}{|l|l|l|l|}
\hline Sr. No. & LO & VPI & Ranking \\
\hline & 36 & 0.1541 & I \\
\hline & 5 & 0.0063 & \\
\hline & 15 & 0.0023 & \\
\hline & 37 & 0.0018 & \\
\hline & 38 & 0.0015 & \\
\hline & 25 & 0.0011 & \\
\hline & 18 & 0.0004 & \\
\hline & 4 & 0.0004 & \\
\hline & 14 & 0.0003 & \\
\hline & 26 & 0.0002 & \\
\hline
\end{tabular}

Table II, presents voltage scenario of IEEE 30-bus system without placement of SVC for base load and for outage of line numbers 36,5 and 15, considering one single line outage at a time. As can be observed from Table II, there is no need of SVC placement for base load without contingency as system is secured from viewpoint of voltage limit violation. 
Voltage Profile Without SVC For Base Load in P.U.

\begin{tabular}{|c|c|c|c|c|}
\hline \multirow[t]{2}{*}{ Bus No. } & \multicolumn{4}{|c|}{ BASE LOAD } \\
\hline & Base Case & $L O 36$ & $L O 5$ & $L O 15$ \\
\hline & 1.0600 & 1.0600 & 1.0600 & 1.0600 \\
\hline & 1.0430 & 1.0430 & 1.043 & 1.0430 \\
\hline & 1.0215 & 1.0201 & 1.0111 & 1.0274 \\
\hline & 1.0129 & 1.0112 & 1.0012 & 1.0199 \\
\hline & 1.0100 & 1.0100 & 0.9323 & 1.0100 \\
\hline & 1.0121 & 1.0115 & 0.9993 & 1.0112 \\
\hline & 1.0034 & 1.0031 & 0.9601 & 1.0029 \\
\hline & 1.0100 & 1.0100 & 1.0100 & 1.0100 \\
\hline & 1.0510 & 1.0461 & 1.0437 & 1.0454 \\
\hline & 1.0444 & 1.0354 & 1.036 & 1.0362 \\
\hline & 1.0820 & 1.08200 & 1.082 & 1.0820 \\
\hline & 1.0574 & 1.0530 & 1.0524 & 1.0097 \\
\hline & 1.0710 & 1.0710 & 1.0710 & 1.0419 \\
\hline & 1.0424 & 1.0353 & 1.0371 & 0.997 \\
\hline & 1.0378 & 1.027 & 1.0317 & 1.0002 \\
\hline & 1.0447 & 1.0382 & 1.0381 & 1.014 \\
\hline & 1.0391 & 1.0309 & 1.0312 & 1.0242 \\
\hline & 1.0279 & 1.0177 & 1.021 & 1.0001 \\
\hline & 1.0253 & 1.0154 & 1.0178 & 1.0035 \\
\hline & 1.0293 & 1.0196 & 1.0215 & 1.0109 \\
\hline & 1.0321 & 1.0182 & 1.0237 & 1.0225 \\
\hline & 1.0327 & 1.0173 & 1.0243 & 1.0227 \\
\hline & 1.0272 & 1.0045 & 1.0202 & 0.9984 \\
\hline & 1.0216 & 0.9835 & 1.0133 & 1.0052 \\
\hline & 1.0189 & 0.9246 & 1.0093 & 1.0124 \\
\hline & 1.0012 & 0.9051 & 0.9915 & 0.9946 \\
\hline & 1.0257 & 0.8999 & 1.0155 & 1.0254 \\
\hline & 1.0107 & 1.0153 & 1.0009 & 1.0088 \\
\hline & 1.0059 & 0.8770 & 0.9955 & 1.0056 \\
\hline & 0.9945 & 0.8637 & 0.9839 & 0.9942 \\
\hline
\end{tabular}




\section{Outage of line no. 36}

As can be observed from Table I, the highest value of VPI (0.1541) is obtained for outage of line no. 36. Therefore, outage of line no. 36 is the most severe line outage. Real coded GA and proposed NSGA-II have been implemented with population size 24 (no. of load buses) for outage of line no. 36. Both algorithms run for 1000 number of generations to determine the optimal location and size of SVC. For the implementation of RCGA in multi-objective function $\mathrm{F}(u, v)$ the values of $w_{1}$ and $w_{2}$ are varied randomly between 0 and 1. Convergence characteristic for RCGA are shown in Fig. 3 and in Fig. 4 for $w_{1}=1$ (power loss only) and $w_{2}=1$ (voltage deviation only). Fig. 5 illustrates the Pareto-optimal front obtained from implementation of RCGA whereas Fig. 6 shows the well distributed Pareto-optimal front generated by proposed NSGA-II. The Pareto-optimal front provides several solutions to multi objective optimization problem (4) and permits the operator to select adequate one. The best compromise solution for optimal values of power loss and voltage deviation as selected using fuzzy based decision maker are summarized in Table III and in Table IV. As can be observed from Table III and Table IV that optimal location for SVC placement as computed with RCGA is bus no. 30 while it is bus no. 27 for NSGA-II. It can also be observed that if minimization of power loss is given higher priory the optimal location is bus no. 30 whereas if minimization of voltage deviation is preferred the optimal location of SVC placement is found to be bus no. 27. The size of SVC computed from RCGA is -0.0702 while it is found to be 0.1173 p.u. with NSGA-II. As can be observed from Fig. 7 that with outage of line no. 36, the voltage magnitude at bus nos. 25, 26, 27, 29 and 30 was below 0.95 p.u., which after placement of SVC increased significantly.

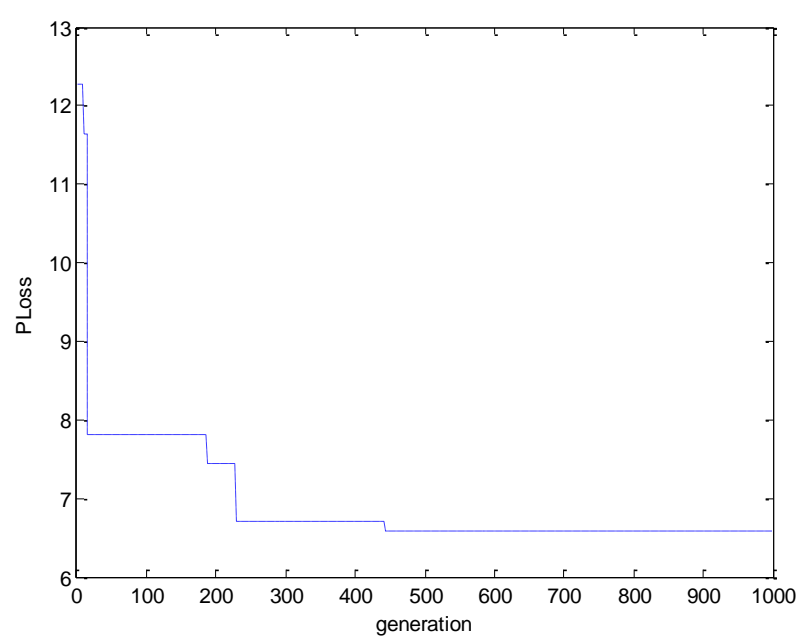

\section{Convergence Characteristic of RCGA for LO 36 when $w_{1}=1$ (Power Loss Only)}




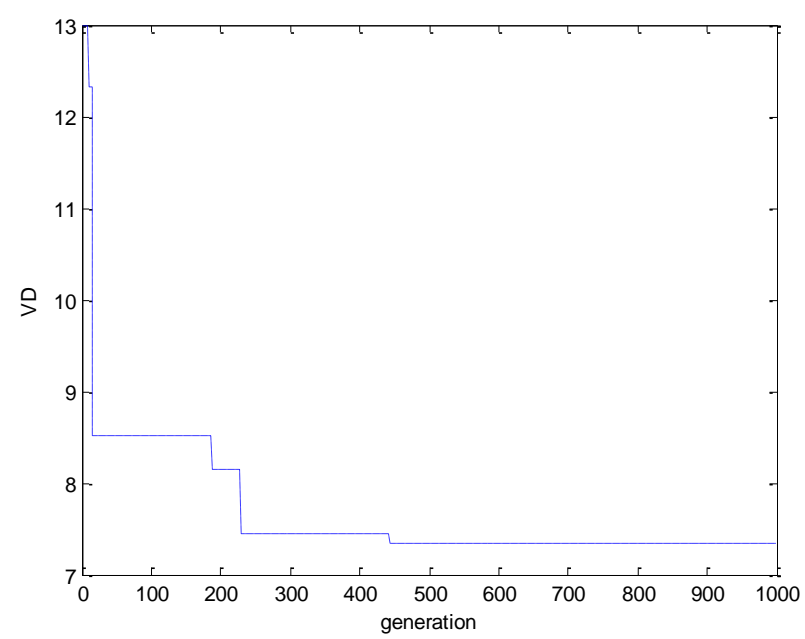

Convergence Characteristic of RCGA for LO 36 when $w_{2}=1$ (Voltage Deviation Only)

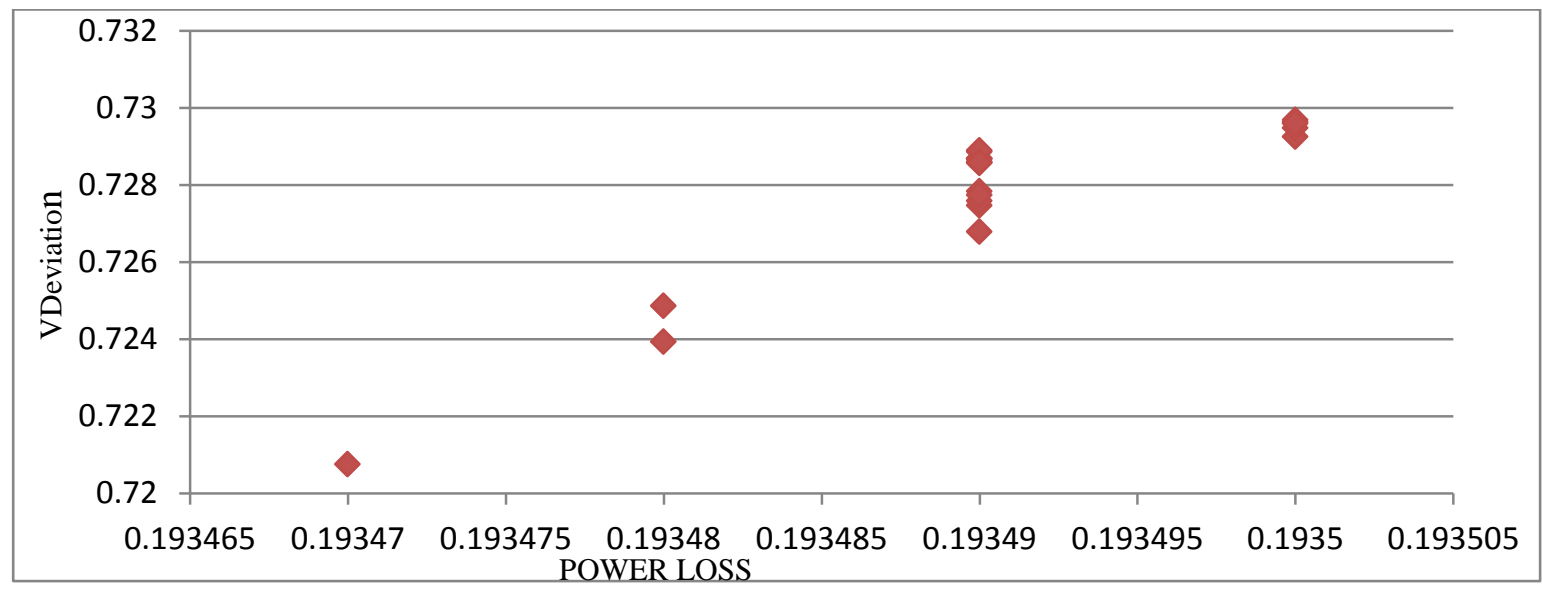

Pareto Front for base Load with LO 36 with RCGA

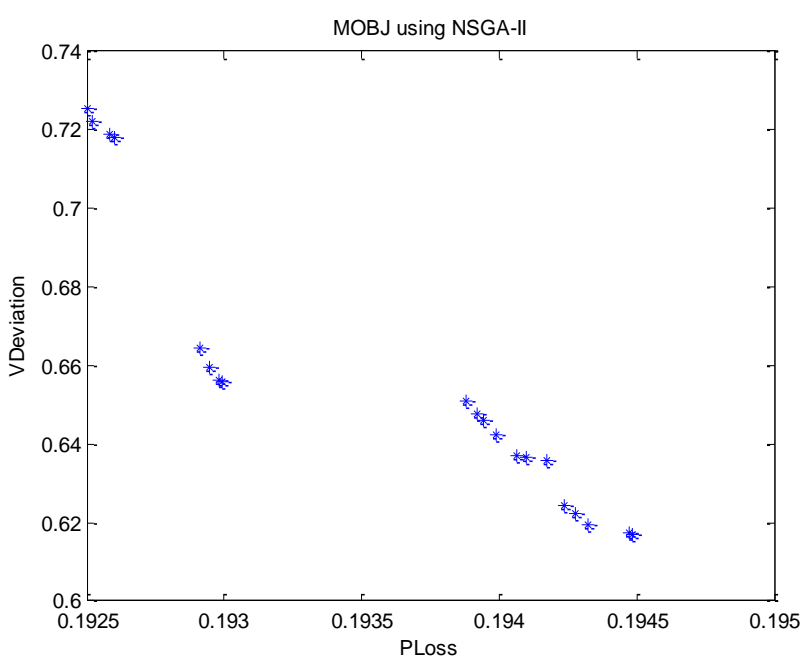

Pareto Front for Base Load with LO 36 with NSGA-II 


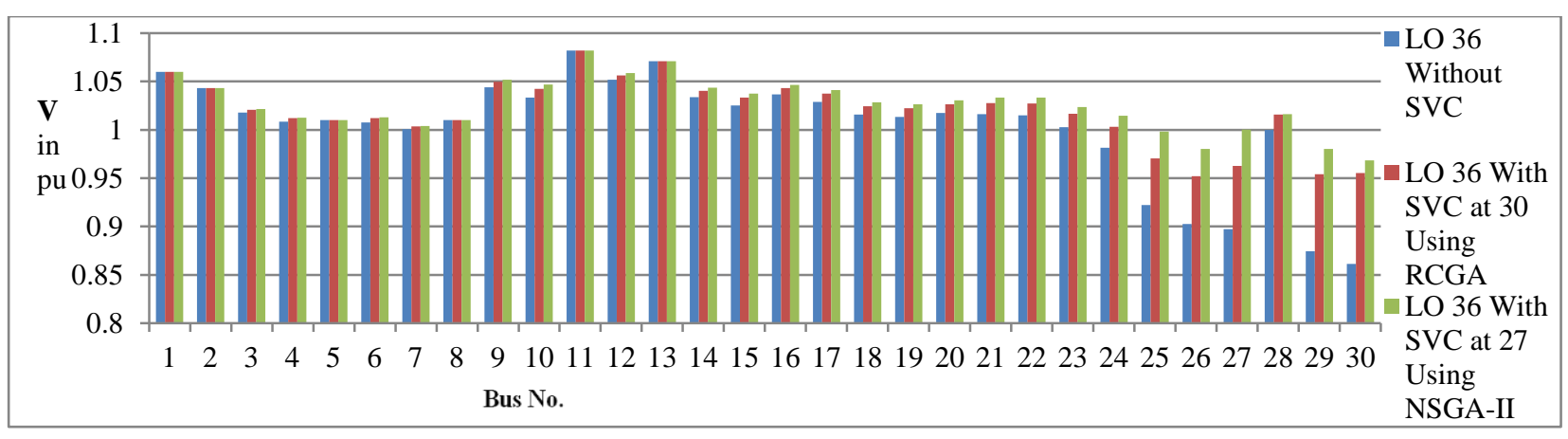

Voltage Profile for Outage of Line no. 36 without and with SVC

Outage of line no. 5

As can be seen from Table 1, the second most severe contingency is outage of line no. 5 with VPI value as 0.0063 . The RCGA and NSGA-II have been applied one by one to find the optimal location and size of SVC following the outage of line no. 5. The number of generations and population size are kept same. The implementation of RCGA to the multiobjective function is done by varying $w_{1}$ and $w_{2}$ randomly between 0 and 1 . The convergence characteristic for RCGA for power loss only $\left(w_{1}=1\right)$ and voltage deviation only $\left(w_{2}=1\right)$ are shown in Fig. 8 and Fig. 9. The Pareto optimal front obtained following outage of line no. 5 with RCGA and NSGA-II are illustrated in Fig 10 and Fig. 11 respectively. As can be observed Fig 10 and Fig. 11 proposed NSGA-II is capable to generate well distributed Pareto optimal front as compare to that obtained by RCGA. The Pareto optimal fronts thus obtained provides several solutions for power loss and voltage deviation minimization. The best compromise solution for optimal values of power loss and voltage deviation as extracted under base case using fuzzy based decision maker is compiled in Table III and Table IV. As can be observed by Table III and Table IV RCGA provides the optimal location as bus no. 12 with minimum voltage deviation while with NSGA-II optimal location of SVC placement is found to be bus no. 20 resulting in minimum power loss. The smaller SVC size is found with RCGA - 0.0932 p.u. as compare to 0.5609 p.u. provided by NSGA-II. The voltage profile before and after placement of SVC is shown in Fig. 11.

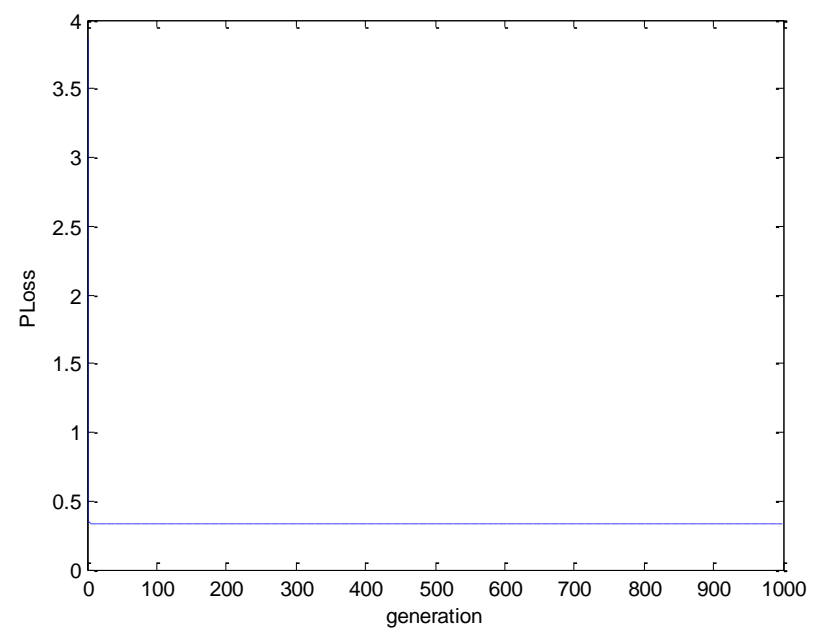

Convergence Characteristic of RCGA for LO 5 when $w_{1}=1$ (power loss only) 


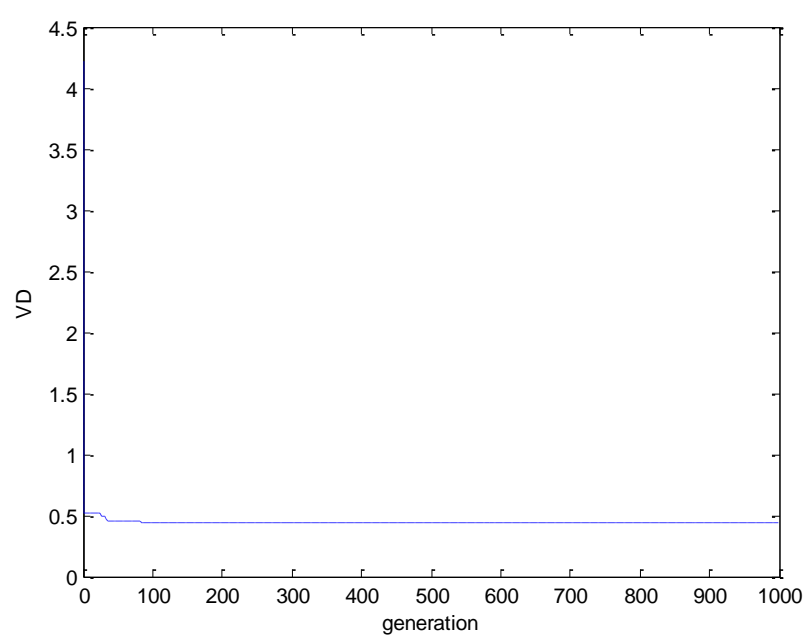

Convergence Characteristic of RCGA for LO 5 when $w_{2}=1$

(Voltage Deviation Only)

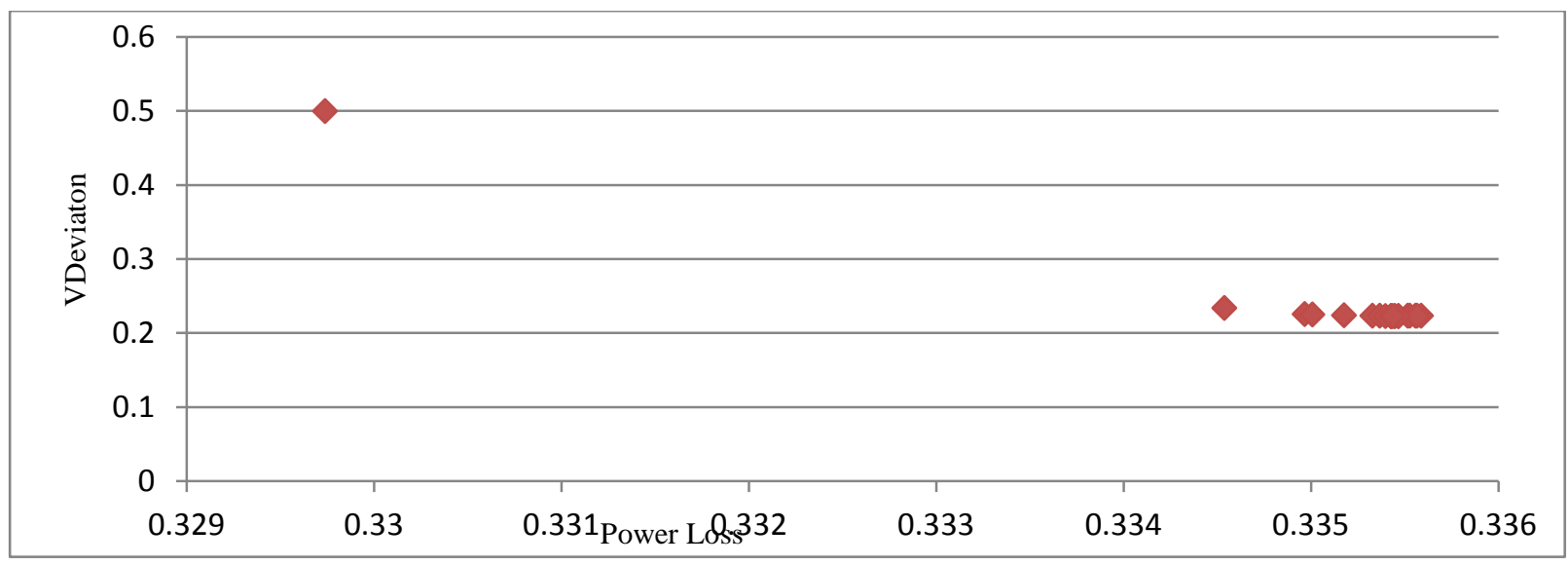

Pareto front Obtained for base Load with LO 5 with RCGA

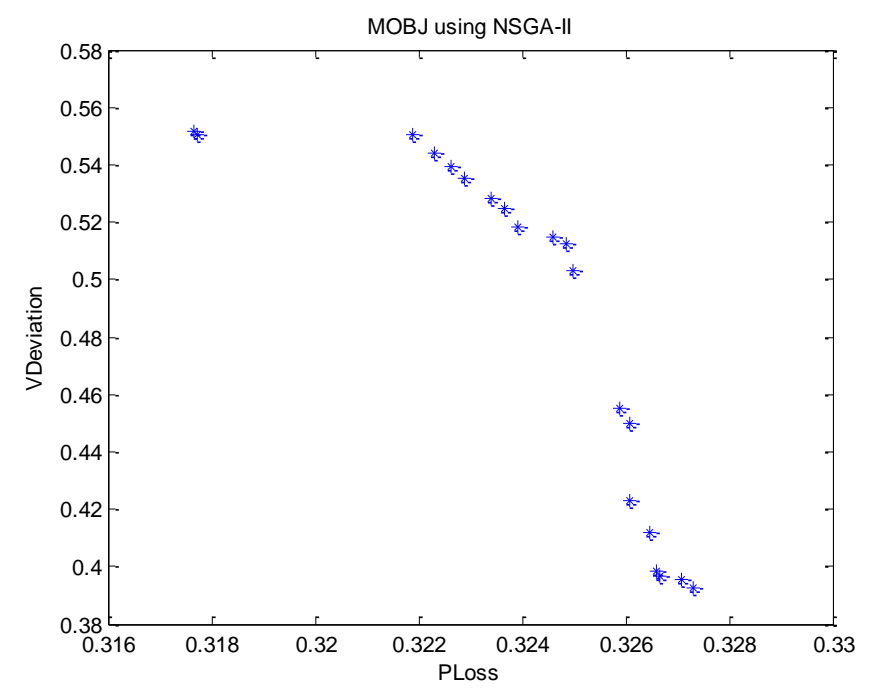

Pareto front for base Load with LO 5 with NSGA-II 


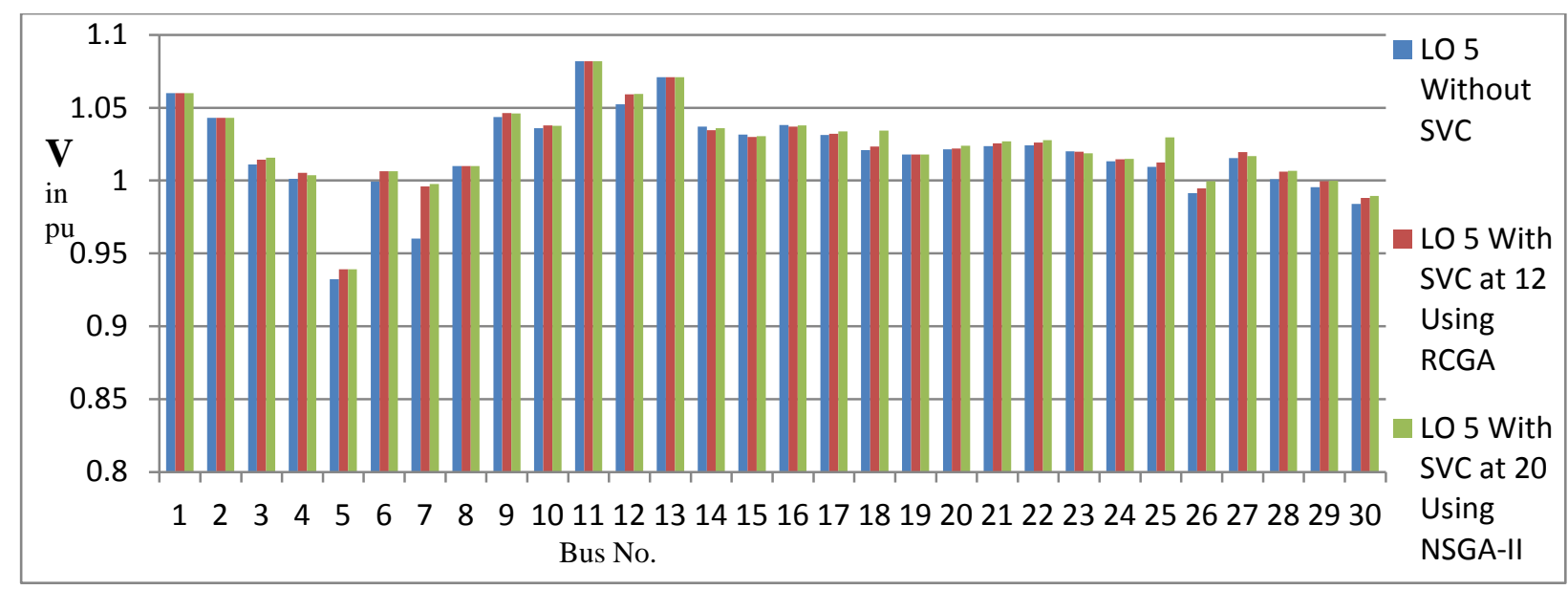

Voltage Profile for Outage of Line no. 5 without and with SVC

Outage of line no. 15

The developed RCGA and NSGA-II have been used for finding optimal location and sizing of SVC following outage of line no. 15 (VPI value as 0.0023 ). The population size and generations are maintained the same. Fig. 13 and Fig. 14 show the convergence characteristic of RCGA for $w_{1}=1$ and for and for $w_{2}=1$ i.e. for power loss only and only for voltage deviation. Fig. 15 and Fig. 16 illustrate Pareto optimal front obtained as a result of RCGA and NGSA-II respectively when line number 15 was out. As can be observed from Fig. 15 and Fig. 16 proposed NSGA-II resulting in well distributed Pareto optimal front in compression of RCGA. The best compromise solution for optimal values of power loss and voltage deviation is selected by using fuzzy decision maker. The best compromise solution obtained for base load with implementation of RCGA and NSGA-II is summarized in Table III and Table IV respectively. As can be observed from Table IV implementation of NSGA-II provides the optimal location of SVC placement is bus no. 21 with size as -0.6613 p.u. resulting in minimum power loss. Execution of RCGA results as bus no. 9 with size 0.5898 p.u. which provides minimum voltage deviation. The Convergence characteristic for RCGA for this case is illustrated in Fig. 16. The voltage magnitude of all the buses with and without SVC is illustrated in Fig. 17.

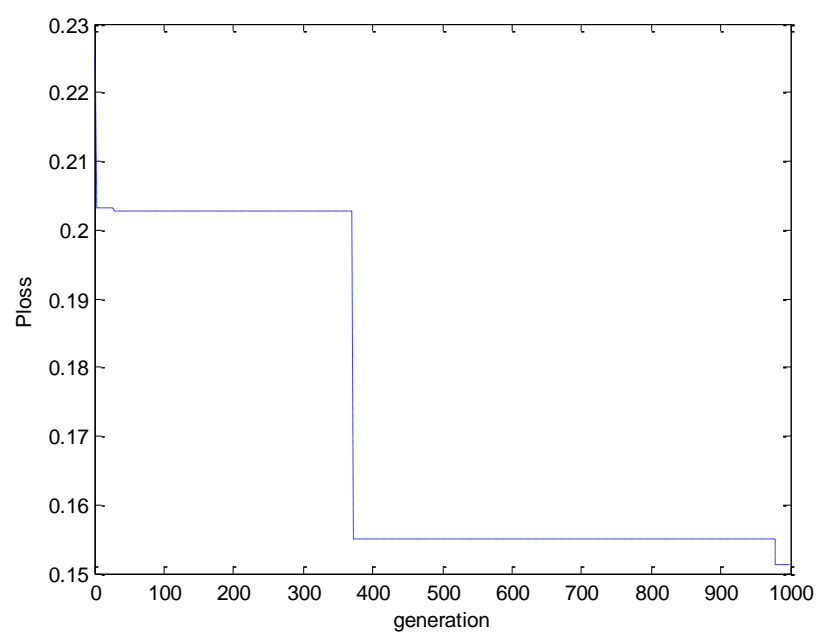

\section{Convergence Characteristic of RCGA for LO 15 when $w_{1}=1$ (Power Loss Only)}




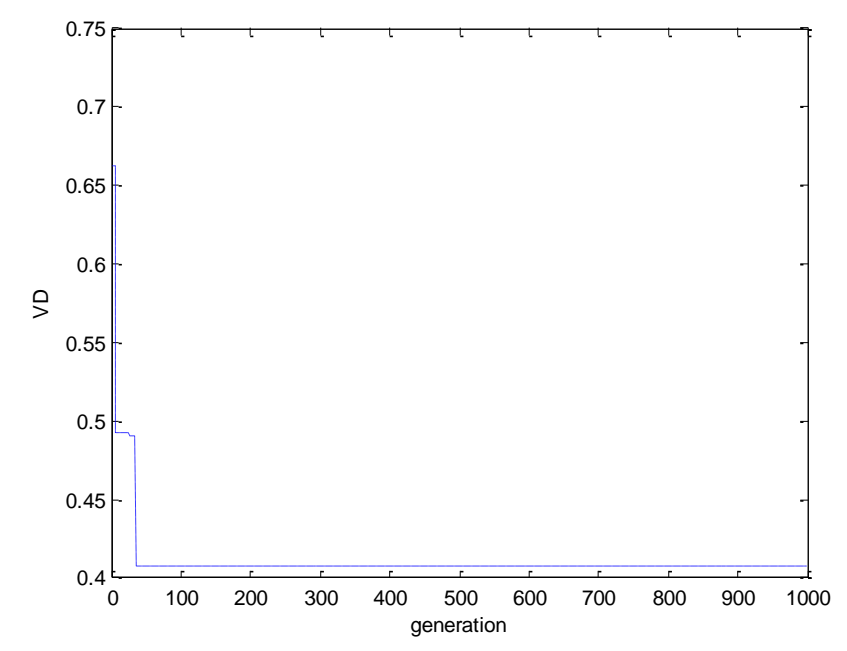

Convergence Characteristic of RCGA for LO 15 when $\mathrm{w}_{2}=1$ (Voltage deviation only)

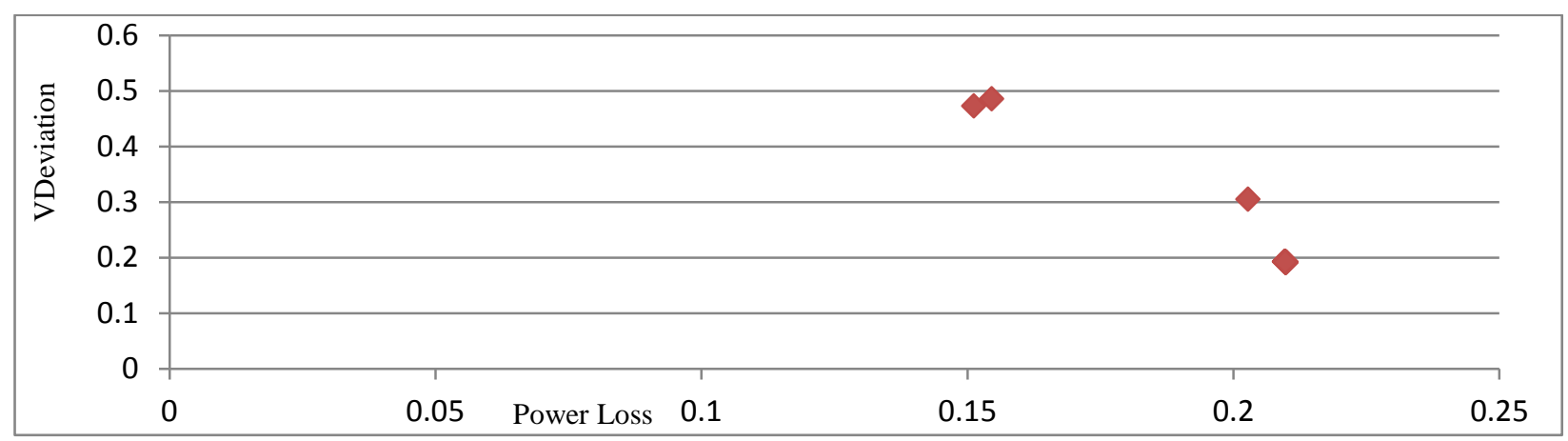

Pareto front Obtained for Base Load with LO 5 with RCGA 


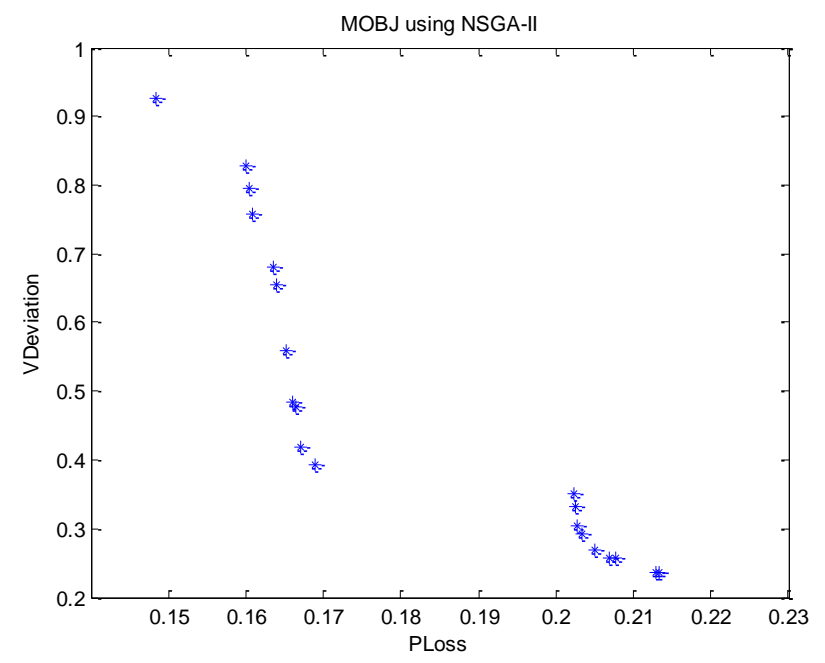

\section{Pareto front for Base Load with LO 15 with NSGA-II}

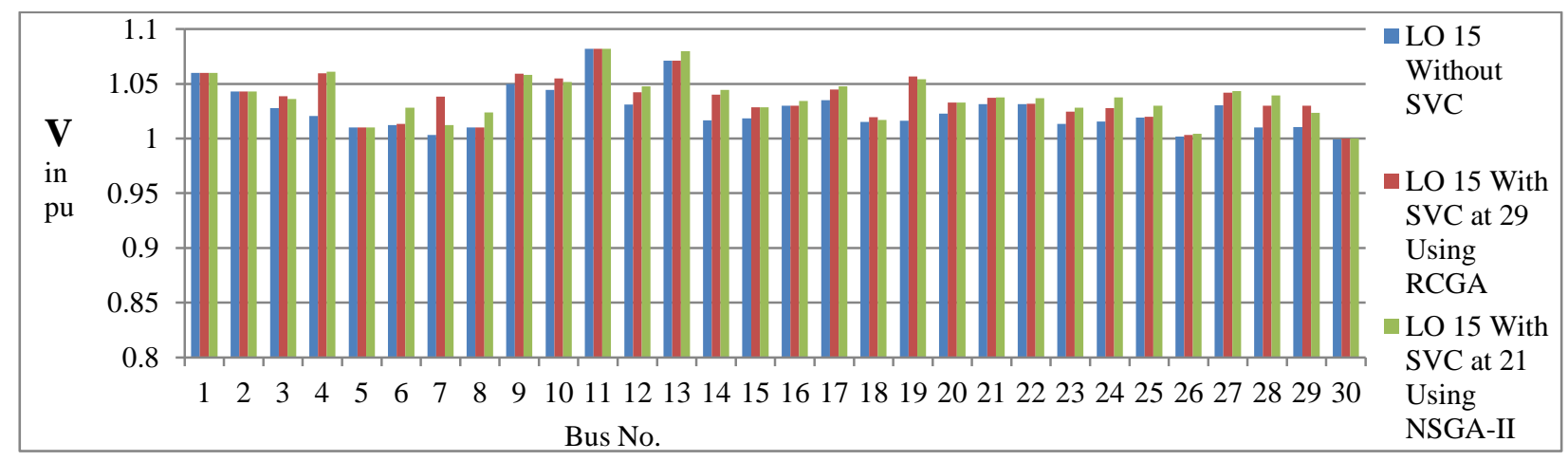

Voltage Profile for Outage of line no. 15 without and with SVC

Best Compromise Result of RCGA

\begin{tabular}{|l|l|l|l|l|}
\hline $\begin{array}{l}\text { Line } \\
\text { Outage }\end{array}$ & $\begin{array}{l}\text { Optimal } \\
\text { Location }\end{array}$ & $\begin{array}{l}\text { Optimal } \\
\text { Size in p.u }\end{array}$ & $\begin{array}{l}\text { Real Power } \\
\text { Loss in p.u }\end{array}$ & $\begin{array}{l}\text { Voltage } \\
\text { Deviation in p.u }\end{array}$ \\
\hline Base case & - & - & 0.1803 & 0.6001 \\
\hline LO 36 & 30 & -0.0702 & 0.1934 & 0.7208 \\
\hline LO 5 & 12 & 0.5609 & 0.3356 & 0.2231 \\
\hline LO 15 & 9 & 0.5898 & 0.2098 & 0.1917 \\
\hline
\end{tabular}

Best Compromise Results of NSGA-II

\begin{tabular}{|l|l|l|l|l|}
\hline $\begin{array}{l}\text { Line } \\
\text { Outage }\end{array}$ & $\begin{array}{l}\text { Optimal } \\
\text { Location }\end{array}$ & $\begin{array}{l}\text { Optimal } \\
\text { Size in p.u }\end{array}$ & $\begin{array}{l}\text { Real Power } \\
\text { Loss in p.u }\end{array}$ & $\begin{array}{l}\text { Voltage } \\
\text { Deviation in p.u }\end{array}$ \\
\hline Base case & - & - & 0.1803 & 0.6001 \\
\hline LO 36 & 27 & 0.1173 & 0.1930 & 0.6562 \\
\hline LO 5 & 20 & -0.0932 & 0.3266 & 0.3966 \\
\hline LO 15 & 21 & -0.6613 & 0.1690 & 0.3924 \\
\hline
\end{tabular}


The implementation of proposed NSGA-II in compression of RCGA provides better solution quality and required less CPU time as it offers results of the multi-optimization in a single run whereas for execution of RCGA to a multi-objective optimization problem required to run in an iterative loop for randomly varying values of $\mathrm{w}_{1}$ and $\mathrm{w}_{2}$ between 0 and 1 . The loop has to run for pre selected set of intervals between randomly varying values of $\mathrm{w}_{1}$ and $\mathrm{w}_{2}$ for the number of generations as decided previously. Thus, execution of RCGA required more computational time in handling truly multi-objective optimization problem.

\section{Conclusion}

In this paper, real coded genetic algorithm (RCGA) and non-dominated sorting genetic algorithm-II (NSGA-II) have been successfully implemented following by outage of critical line to obtain the optimal location and size of SVC to minimize real power loss and load bus voltage deviation. The problem is formulated as multi-objective optimization. It has been concluded that optimal placement of SVC can enhance voltage security significantly in a power system. Implementation of RCGA and NSGA-II on IEEE 30-bus test system indicates that proposed methods are capable to provide the optimal location and size of SVC. From the results obtained it can also be concluded that NSGAII is more effective in providing results of multi-objective optimization in a single run with better solution quality and in less computational effort as well as in less computational computational time. NSGA-II is also found to be more capable for generating well distributed Pareto-optimal front. Though, the proposed approaches have been implemented on IEEE 30-bus test system, the same can be implemented on practical power system as well.

\section{Acknowledgement}

The authors sincerely acknowledge the financial support provided by University Grants Commission (UGC), New Delhi, India under Major Research Project received vide F. No. 41-657/2012-SR dated 26-07-12 and the Director, MITS Gwalior, India to carry out this work.

\section{References}

[1] Kazemi, B. Badrzadeh, Modeling and simulation of SVC and TCSC to study their limits on maximumloadability point, Electrical Power and Energy Systems 26 (April) (2004) 619-626.

[2] J.R. Shin, B.S. Kim, J.B. Park, K.Y. Lee, A new optimal routing algorithm for loss minimization and voltage stability improvement in radial power systems, IEEE Transactions on Power Systems 22 (May (2)) (2007) 648-657.

[3] M.M. Farsangi, H. Nezamabadi-pour, Y.-H. Song, K.Y. Lee, Placement of SVCs and selection of stabilizing signals in power systems, IEEE Transactions on PowerSystems 22 (August (3)) (2007) 10611071.

[4] Hsiao Y.T., Liu C.C., Chiang H.D., A new approach for optimal VAR sources planning in large scale electric power systems,IEEE Trans. Power Syst, 8 (1993) , 988-996.

[5] S.N. Singh, Electrical Power Generation, Transmission and Distribution, PHI Leaning Pvt. Ltd. , 2004

[6] G. S. Dohare and L. Srivastava, "Optimal placement and sizing of TCSC for minimization of line overloading and system power loss using multi-objective genetic algorithm," Int. Conf. on Advanced Engg. Optimization Through Intelligent Techniques,AEOTIT, pp. 470-476, Surat, India, 2013.

[7] D. V. M. Chary and J. Amarnath, "Complex neural network approach to optimal location of FACTS devices for transfer capability enhancement," APRN Journal of Engineering and Applied Sciences, , Vol 5, pp. 21-25, January 2010.

[8] M. A. Kamarposhti, M. Alinezhad, H. Lesani and N. Talebi, "Comparison of SVC, STATCOM, TCSC and UPFC controllers for static voltage stability evaluated by continuation power flow method," IEEE Electric Power Conference, EPEC, pp. 1-8, Canada 2008.

[9] M. Saravanan, S.M.R. Slochanal, P. Venkatesh, J. Prince Stephen Abraham, Application of particle swarm optimization technique for optimal location of FACTS. devices considering cost of installation and system loadability, Electric Power System Research 77 (2007) 276-283. 
[10] M.M. Farsangi, H. Nezamabadi-Pour, K.Y. Lee, Multi-objective VAR planning with SVC for a large power system using PSO and GA, in: Proceeding of Power System Conference \& Exposition, 2006, pp. 274-279.

[11] Y. Mansour, W. Xu, F. Alvarado, C. Rinzin, SVC placement using critical modes of voltage instability, IEEE Transactions on Power Systems 9 (May (2)) (1994) 757-763.

[12] L.A.S. Pilotto, et al., Determination of needed facts controllers that increase asset utilization of power systems, IEEE Transactions on Power Delivery 12 (January (1)) (1997) 364-371.

[13] S.N. Singh, A.K. David, Placement of facts devices in open power market, in:Proceedings of the 5th International Conference on Advances in Power System Control, Operation and Management, APSCOM2000, HongKong, October, 2000, pp. 173-177.

[14] M.K. Verma, S.C. Srivastava, Optimal placement of SVC for static and dynamic voltage security enhancement, International Journal ofEmerging Electric Power Systems 2 (2) (2005) (Article 1050).

[15] A.K. Sharma, Optimal number and location of TCSC and loadability enhancement in deregulated electricity markets using MINLP, International Journal of Emerging Electric Power Systems 5 (1) (2006) (Article 6).

[16] S. Gerbex, R. Cherkaoui, A.J. Germond, Optimal location of multi-type FACTS devices in power system by means of genetic algorithm, IEEE Transactions on Power Systems 16 (August (3)) (2001) 2001.

[17] L. Cai, I. Erlich, G. Stamtsis,Y. Luo,Optimal choice and allocation of FACTS devices in deregulated electricity market using genetic algorithms, Power SystemsConference and Exposition 1 (October (1013)) (2004) 201-207.

[18] L. Ippolito, A.L. Cortiglia, M. Petrocelli, Optimal allocation of FACTS devices by using multi-objective optimal power flowand genetic algorithms, International Journal of Emerging Electric Power Systems 7 (2) (2006) (Article 1).

[19] Minguez R., Milano F., Zarate-Minano R., Conejo A., Optimal Network Placement of SVC Devices, IEEE Trans. Power Syst, 22 (2007), No. 4,1851-1861.

[20] Singh J.G., Singh S. N., Srivastava S. C., An Approach for Optimal Placement of Static VAR Compensators Based on Reactive Power Spot Price, IEEE Trans. Power Syst,22 (2007), No.4, 2021 2029.

[21] Hsiao Y.T., Liu C.C., Chiang H.D., A new approach for optimal VAR sources planning in large scale electric power systems,IEEE Trans. Power Syst, 8 (1993), 988-996.

[22] Ishak S., Abidin A.F., Rahinan T.K.A.,Static Var Compensator Planning Using Artificial Immune System For Loss Minimisation And Voltage Improvement, National Power \& EnergyConference (PECon) 2004 Proceedings, KuaIa Lumpur,Malaysia,(2004), 41-46.

[23] R. Narmatha Banu, D. Devaraj, 'Multi-objective GA with fuzzy decision making for security enhancement in power system'. Applied Soft Computing 12 (2012) 2756-2764.

[24] Kazemi A., Parizad A. , Baghaee H., On the use of harmony search algorithm in optimal placement of FACTS devices to improve power system security, Proceedings of the IEEE Eurocon,Tehran, Iran, (2009),570-576.

[25] Reza Sirjani, Azah Mohamed, Hussain Shareef, “Optimal allocation of shunt Var compensators in power systems using novel global harmony search algorithm” Electrical Power and Energy Systems, 43 (2012), $562-572$.

[26] S. Sakthivel and D. Mary, "Big Bang-Big Crunch Algorithm for Voltage Stability Limit Improvement by Coordinated Control of SVC Settings" Research Journal of Applied Sciences, Engineering and Technology 6(7) (2013), 1209-1217.

[27] Dixit S, Srivastava L, Agnihotri, G., "Optimal placement of SVC for minimizing power loss and improving voltage profile using GA" in International Conference on Issues and Challenges in Intelligent Computing Techniques (ICICT), 2014, pp 123-129.

[28] Srivastava L, Dixit S, Agnihotri, Ganga, "Optimal location and size of TCSC for voltage stability enhancement using PSO-TVAC" in International Conference on Power and Energy Systems Conference: Towards Sustainable Energy, 2014, pp 1-6.

[29] Allen J.Wood, Bruce F.Wollenberg, Power Generation Operation and Control, IIEd., John Willey \& Sons, Inc, 2004.

[30] Herrera F, Lozano M, Verdegay JL. Tackling real-coded genetic algorithms: operators and tools for behavioral analysis, Artificial Intelligence Review 12, Kluwer Academic Publishers, 1998, p. 265-319.

[31] S. N. Sivanandam, S. N. Deepa, Introduction to Genetic Algorithms, Springer Berlin Heidelberg New York, 2008.

[32] Deb K. Multi-Objective Optimization using Evolutionary Algorithms John Wiley \& Sons, Ltd.; 2002.

[33] E. Eiben, J. E. Smith, Introduction to Evolutionary Computing, Springer, 2003.

[34] Deb K, Agrawal RB. Simulated binary crossover for continuous search space. Complex Syst 1995;9 (2): $115-48$.

[35] Herrera F, Lozano M, Verdegay JL. Tackling real-coded genetic algorithms: operators and tools for behavioral analysis, Artificial Intelligence Review 12, Kluwer Academic Publishers, 1998, p. 265-319.

[36] M. Basu, "Dynamic economic emission dispatch using nondominated sorting genetic algorithm-II" Electrical Power and Energy Systems 30 (2008) 140-149. 
International Journal of Grid and Distributed Computing

Vol. 9, No. 9 (2016)

[37] Hadi Saadat, Power System Analysis, Tata McGRAW-HILL Edition. 